\title{
ASASSN-18aan: An Eclipsing SU UMa-type Cataclysmic Variable with a 3.6-hour Orbital Period and a Late G-type Secondary Star
}

Yasuyuki Wakamatsu, ${ }^{1 *}$ John R. Thorstensen, ${ }^{2}$ Naoto KoJIGUCHI, Keisuke Isogal, ${ }^{1,3}$ Mariko KImura, ${ }^{1,4}$ Ryuhei OHnishi, ${ }^{1}$ Taichi Kato, ${ }^{1}$ Hiroshi ITOH, ${ }^{5}$ Yuki Sugiura, ${ }^{6}$ Sho SumiYa, ${ }^{6}$ Hanami Matsumoto, ${ }^{6}$ Daiki ITo, ${ }^{6}$ Kengo Nikal, ${ }^{6}$ Hiroshi AKITAYA, ${ }^{7,8}$ Chihiro IsHIOKA, ${ }^{9}$ Kohei OIDE, ${ }^{9}$ Takahiro KanAI, ${ }^{9}$ Yoshinori UzawA, ${ }^{10}$ Yumiko OASA, ${ }^{7,9,10}$ Tamás Tordal, ${ }^{11}$ Tonny Vanmunster, ${ }^{12}$ Sergey Yu. Shugarov, ${ }^{13,14}$ Masayuki YamanaKa, ${ }^{3,8}$ Mahito Sasada, ${ }^{8}$ Kengo TaKagl, ${ }^{8}$ Yuki NishinaKa, ${ }^{8}$ Yuina YamAZAKI, ${ }^{8}$ Ikki OTsubo, ${ }^{8}$ Tatsuya NAKAOKA, ${ }^{8}$ Katsuhiro L. Murata, ${ }^{15}$ Ryou OHsawa, ${ }^{16,17}$ Masahiro Morita, ${ }^{16}$ Makoto ICHIKI, ${ }^{16}$ Sjoerd DUfOER, ${ }^{18}$ Masanori MIZUTANI, ${ }^{19}$ Takashi HoRIUCHI, ${ }^{20}$ Miyako TozUKA, ${ }^{21}$ Masaki TAKAYAmA, ${ }^{21}$ Tomohito OHshima, ${ }^{21}$ Tomoki SaIto, ${ }^{21}$ Pavol A. Dubovsky, ${ }^{22}$ Geoff Stone, ${ }^{23}$ lan MILLER, ${ }^{24}$ and Daisaku NogAmi ${ }^{1}$

${ }^{1}$ Department of Astronomy, Kyoto University, Kyoto 606-8502, Japan

2 Department of Physics and Astronomy, 6127 Wilder Laboratory, Dartmouth College, Hanover, NH 03755-3528, USA

${ }^{3}$ Okayama Observatory, Kyoto University, 3037-5 Honjo, Kamogatacho, Asakuchi, Okayama 719-0232, Japan

${ }^{4}$ Extreme Natural Phenomena RIKEN Hakubi Research Team, Cluster for Pioneering Research, RIKEN, 2-1 Hirosawa, Wako, Saitama 351-0198, Japan

${ }^{5}$ Variable Star Observers League in Japan (VSOLJ), 1001-105 Nishiterakata, Hachioji, Tokyo 192-0153, Japan

${ }^{6}$ Osaka Kyoiku University, 4-698-1 Asahigaoka, Kashiwara, Osaka 582-8582, Japan

${ }^{7}$ Graduate School of Science and Engineering, Saitama University, 255 Shimo-Okubo, Sakura-ku, Saitama, Saitama 338-8570, Japan

8 Hiroshima Astrophysical Science Center, Hiroshima University, 1-3-1 Kagamiyama, Higashi-Hiroshima, Hiroshima 739-8526, Japan

${ }^{9}$ Graduate School of Education, Saitama University, 255 Shimo-Okubo, Sakura-ku, Saitama, Saitama 338-8570, Japan

${ }^{10}$ Faculty of Education, Saitama University, 255 Shimo-Okubo, Sakura-ku, Saitama, Saitama 338-8570, Japan

11 Polaris Observatory, Hungarian Astronomical Association, Laborc utca 2/c, 1037 Budapest, Hungary

12 Center for Backyard Astrophysics Belgium, Walhostraat 1A, B-3401 Landen, Belgium

${ }^{13}$ Sternberg Astronomical Institute, Lomonosov Moscow State University, Universitetsky Ave., 13, Moscow 119992, Russia

${ }^{14}$ Astronomical Institute of the Slovak Academy of Sciences, 05960 Tatranska Lomnica, Slovakia 
15 Department of Physics, Tokyo Institute of Technology, 2-12-1 Ookayama, Meguro-ku, Tokyo 152-8551, Japan

${ }^{16}$ Institute of Astronomy, Graduate School of Science, The University of Tokyo, 2-21-1

Osawa, Mitaka, Tokyo 181-0015, Japan

17 Kiso Observatory, Institute of Astronomy, Graduate School of Science, The University of

Tokyo 10762-30, Mitake, Kiso-machi, Kiso-gun, Nagano 397-0101, Japan

18 Vereniging Voor Sterrenkunde (VVS), Oostmeers 122 C, 8000 Brugge, Belgium

${ }^{19}$ Variable Star Observers League in Japan (VSOLJ), Okayama, Japan

20 Ishigakijima Astronomical Observatory, Public Relations Center, National Astronomical

Observatory of Japan, 1024-1 Arakawa, Ishigaki, Okinawa, 907-0024, Japan

${ }^{21}$ Nishi-Harima Astronomical Observatory, Center for Astronomy, University of Hyogo, 407-2,

Nishigaichi, Sayo-cho, Sayo, Hyogo 679-5313, Japan

22 Vihorlat Observatory, Mierova 4, 06601 Humenne, Slovakia

${ }^{23}$ American Association of Variable Star Observers, 49 Bay State Rd., Cambridge, MA 02138, USA

${ }^{24}$ Furzehill House, Ilston, Swansea, SA2 7LE, UK

*E-mail: *wakamatsu@kusastro.kyoto-u.ac.jp

Received 201 0; Accepted 2010

\section{Abstract}

We report photometric and spectroscopic observations of the eclipsing SU UMa-type dwarf nova ASASSN-18aan. We observed the 2018 superoutburst with 2.3 mag brightening and found the orbital period $\left(P_{\text {orb }}\right)$ to be $0.149454(3) \mathrm{d}$, or $3.59 \mathrm{hr}$. This is longward of the period gap, establishing ASASSN-18aan as one of a small number of long- $P_{\text {orb }}$ SU UMa-type dwarf novae. The estimated mass ratio, $\left(q=M_{2} / M_{1}=0.278(1)\right)$, is almost identical to the upper limit of tidal instability by the 3:1 resonance. From eclipses, we found that the accretion disk at the onset of the superoutburst may reach the 3:1 resonance radius, suggesting that the superoutburst of ASASSN-18aan results from the tidal instability. Considering the case of long- $P_{\text {orb }}$ WZ Sge-type dwarf novae, we suggest that the tidal dissipation at the tidal truncation radius is enough to induce SU UMa-like behavior in relatively high- $q$ systems such as SU UMatype dwarf novae, but that this is no longer effective in low- $q$ systems such as WZ Sge-type dwarf novae. The unusual nature of the system extends to the secondary star, for which we find a spectral type of G9, much earlier than typical for the orbital period, and a secondary mass $M_{2}$ of around $0.18 \mathrm{M}_{\odot}$, smaller than expected for the orbital period and the secondary's spectral type. We also see indications of enhanced sodium abundance in the secondary's spectrum. Anomalously hot secondaries are seen in a modest number of other CVs and related objects. These systems evidently underwent significant nuclear evolution before the onset of mass transfer. In the case of ASASSN-18aan, this apparently resulted in a mass ratio lower than typically found at the system's $P_{\text {orb }}$, which may account for the occurrence of a superoutburst at this relatively long period.

Key words: accretion, accretion disks - stars: novae, cataclysmic variables — stars: dwarf novae stars: individual (ASASSN-18aan)

\section{Introduction}

Cataclysmic variables (CVs) are close binary systems consisting of a white dwarf primary star and a secondary star that fills its Roche lobe and transfers mass to the primary via the inner Lagrangian point $L_{1}$. Unless the white dwarf is highly magnetized, an accretion disk forms around the primary. Dwarf novae (DNe) are a subclass of CVs characterized by recurrent outbursts of 2-10 magnitude, evidently caused by accretion disk instabilities. 
Over most of the life of a $\mathrm{CV}$, the binary separation gradually decreases due to angular momentum loss from the system, resulting shortening of the orbital period. The mass ratio also decreases due to the mass transfer from the secondary to the primary. CVs therefore generally evolve toward extreme states with low mass ratios and short orbital periods. Relatively few non-magnetic CVs are observed in a period range from roughly 2 to $3 \mathrm{hr}$, known as the period gap (see Knigge et al. 2011 and references therein for more on $\mathrm{CV}$ evolution).

SU UMa-type DNe, a subclass of DNe, show longlasting, brighter outbursts called superoutbursts in addition to (normal) outbursts. It is a key feature of superoutbursts to be accompanied by superhumps, which are variations of small amplitude, typically 0.1-0.5 mag, and have slightly longer periods than orbital periods. The superoutburst is considered to be a result of the tidal instability that is triggered when the outer disk reaches the 3:1 resonance radius (Whitehurst 1988; Hirose, Osaki 1990; Lubow 1991; Lubow 1991). The expansion of the accretion disk to the $3: 1$ resonance radius can only occur if the mass ratio $q=M_{2} / M_{1}$ is less than 0.25 , (Whitehurst 1988), or 0.33 with reduction of the mass transfer from the secondary (Murray et al. 2000). The period of most SU UMa-type DNe lie below the period gap; at these short periods, the mass rations are typically $<0.25$ The expansion of the accretion disk is limited by the tidal truncation radius, where the angular momentum of orbiting material is removed via tidal torque. The $3: 1$ resonance, therefore, is considered to occur when the tidal truncation radius is larger than the 3:1 resonance radius. The detailed action of the tidal truncation radius, however, is still unknown and it is unclear whether it acts as a hard limit of disk expansion, especially in extremely low- $q$ systems (Osaki, Meyer 2002).

A small number of CVs with longer periods have shown the superhumps and superoutbursts characteristic of SU UMa stars; these long- $P_{\text {orb }}$ objects should offer tests of the tidal instability model and the effects of tidal torques at the tidal truncation radius.

The longest-known of these long-period SU UMa stars is TU Men (Stolz, Schoembs 1984), which has an orbital period $P_{\text {orb }}=0.1172(2)$, or $2.81 \mathrm{hr}$ (Mennickent 1995). The mass ratio of TU Men is estimated by various authors; 0.58 (Stolz, Schoembs 1984), 0.455(45) or 0.33 (Mennickent 1995), 0.5(2) (Smak 2006). Smak (2006) also presented the lower limit of $q>0.41(8)$. TU Men has been treated as an exceptional SU UMa-type DN because of its long orbital period and anomalously large mass ratio, which is far beyond the upper critical value of the 3:1 resonance predicted by the tidal instability model.

Other SU UMa-type DNe have been detected in the pe- riod gap (see, e.g., Katysheva, Pavlenko (2003), Pavlenko et al. (2014)). More recently, some SU UMa-type DNe have been discovered above the period gap. In 2018, CS Ind (Kato et al. 2019a) showed the superoutburst accompanied by superhumps with the mean superhump period $P=0.12471(1) \mathrm{d}$ and the behavior of the superoutburst is similar to that of WZ Sge-type DNe, which is a subclass of SU UMa-type DNe and have extremely short orbital periods and small mass ratios (see Kato (2015) for a review). OT J002656.6+284933 showed superoutburst in 2016 and has an unlikely small mass ratio, less than 0.15 , in spite of its long superhump period of $0.13225(1)$ d (Kato et al. 2017). ASASSN-14ho is another curious SU UMa-type DNe. This object showed four rebrightenings after the main superoutburst in 2014 (Kato 2020). The orbital period and mass ratio are $0.24315(10) \mathrm{d}$ and 0.28, respectively (Gasque et al. 2019). This orbital period is the longest one among known SU UMa-type DNe. Additionally, the secondary of ASASSN-14ho is anomalously warm for its orbital period; its spectral type is K4 \pm 2 although the expected type from the standard model (Knigge 2006) is M0 (Gasque et al. 2019). These CVs with anomalously hot secondaries are considered to be formed when the secondary underwent significant nuclear evolution prior to the onset of the mass transfer (Thorstensen 2015).

It should be noted that Smak, Waagen (2004) reported the detection of superhumps in the 1985 long outburst of U Gem. The orbital period and mass ratio of U Gem are 0.1769061911(28) (Marsh et al. 1990) and 0.364(17) (Smak 2001), respectively. If U Gem indeed underwent a superoutburst, this presents a serious challenge to the tidal instability model since the mass ratio of $U$ Gem is too large to grow tidal instability.

In addition to long- $P_{\text {orb }}$ SU UMa-type DNe, some WZ Sge-type DNe have been reported that have atypically long orbital periods. ASASSN-16eg (Wakamatsu et al. 2017), which showed superoutburst accompanied by clear early superhumps, has long orbital period and anomalously large mass ratio, almost twice the upper limit of the 2:1 resonance. Osaki, Meyer (2002) suggested that in extremely low $q$-systems such as WZ Sge-type DNe, the tidal torque by the secondary at the tidal truncation radius is weak and the outer disk could reach the 2:1 resonance radius beyond the tidal truncation radius.

In this paper, we report observations of ASASSN-18aan $\left(\alpha=0^{\mathrm{h}} 46^{\mathrm{m}} 08^{\mathrm{s}} \cdot 05, \delta=+62^{\circ} 10^{\prime} 04^{\prime \prime} .9\right.$, from the Gaia Collaboration et al. 2018). The superoutburst was detected on 2018 November 30 by the All-Sky Automated Survey for Supernovae (ASAS-SN: Shappee et al. 2014). The inverse of the Gaia DR2 parallax is $675(+32,-29)$ 
pc (Gaia Collaboration et al. 2018). Green et al. (2018) computed three-dimensional reddening maps across the northern sky ${ }^{1}$. Their map gives $E(g-r)=0.35$ at the Gaia distance along this line of sight; using expressions found in Green et al. (2019) we convert this to $A_{V}=1.19$ mag, or $E(B-V)=0.36 \mathrm{mag}$, where we have assumed $R=A_{V} / E(B-V)=3.32$. Nesci et al. (2019) obtained an optical spectrum indicating the spectral type of the secondary is $\mathrm{G}-\mathrm{K}$. They also investigated historical outbursts of ASASSN-18aan from plates and found that the recurrence time may be about 11 months. The object showed superhump-like modulations in the 2018 superoutbursts, as well as clear eclipses. The eclipses revealed on orbital period that was quite long for an SU UMa-type DN, 0.1495 $\mathrm{d}$ as a tentative value, which attracted special interest (vsnet-alert 22806, 22810). Observations showed the superhump period to be much longer than the orbital one, indicating an unusually large mass ratio (vsnet-alert 22816, 22817,22821 ). We therefore performed world-wide photometric and spectroscopic observations. We also performed photometric and spectroscopic observations in quiescent state to clarify its nature and binary parameters. Our observations are described in section 2 , and their results are in section 3. We describe the analysis in section4, and discuss the nature of this anomalous object in section 5 . We summarize our conclusions in section 6 .

\section{Observations}

\subsection{Spectroscopy}

Our most extensive set of spectra is from the Ohio State Multi-Object Spectrograph (OSMOS; Martini et al. 2011) mounted on the $2.4 \mathrm{~m}$ Hiltner telescope at MDM Observatory, on Kitt Peak, Arizona. We obtained a sequence of eighteen $720 \mathrm{~s}$ exposures of ASASSN-18aan on 2019 Sept. 06 UT. The spectra span $4.12 \mathrm{hr}$ from start to finish, and cover just over one orbit. The spectra cover from 3975 to $6890 \AA$, with $0.7 \AA$ pixel $^{-1}$ and $3.0 \AA$ resolution (full width at half-maximum). We derived a pixel-towavelength relation from $\mathrm{Hg}, \mathrm{Ne}$, and Xe lamp spectra, and used the $[\mathrm{OI}]$ and $\mathrm{OH}$-band features in the night sky spectrum to derive zero point offsets and linear stretch factors that we applied to the wavelength scales of the individual exposures. To convert to absolute flux, we observed spectrophotometric standard stars in twilight when the sky was reasonably clear.

\footnotetext{
${ }^{1}$ One can query these maps at http://argonaut.skymaps.info/
}

\subsection{MDM Time Series Photometry}

Our MDM time series photometry (Table 2) is all from the McGraw-Hill 1.3m telescope. In 2019 September we used an Andor frame-transfer camera on three nights; exposures were generally 30 seconds with almost no dead time between exposures. In 2019 October we used a SITe $1024^{2}$ CCD detector cropped to $256^{2}$, which resulted in a $\sim 3$ s dead time. For most of the observations we used a Schott GG420 glass filter, which blocks $\lambda<4200 \AA-$ functionally similar to no filter - but for one night in September we used an $r$ filter. The same set of comparison stars was used througout. The main comparison star, at $\alpha=0^{\mathrm{h}} 46^{\mathrm{m}} 05^{\mathrm{s}} .8, \delta=+62^{\circ} 10^{\prime} 43^{\prime \prime} .4(\mathrm{~J} 2000)$, is listed at $V=14.446$ in APASS (Henden et al. 2011). We adjusted our differential magnitudes by this amount to convert them to rough $V$ magnitudes.

\subsection{VSNET \& OISTER Time Series Photometry and spectroscopy}

We performed a world-wide observational campaign via Variable Star Network (VSNET) collaborations (Kato et al. 2004) and Optical and Infrared Synergetic Telescopes for Education and Research (OISTER). All of our photometric observations by VSNET and OISTER were described in barycentric Julian date (BJD). Detailed logs of photometric observations by VSNET and OISTER are listed in table E2. Our intensive observations of the superoutburst of ASASSN-18aan by VSNET was started on December 4, 2018 (BJD 2458457), 4 days after the ASASSN's detection.

We also performed spectroscopic observations via OISTER to confirm changes of spectral features through the superoutburst on 2018-12-10, 2018-12-24 and 2019-0105 (UT). We could not observed standard stars due to a bad weather and time limitation so not performed a sensitivity correction.

Table 1 gives a summary of the OISTER observations.

\section{Results}

\subsection{Overall light curve of superoutburst}

The overall light curve of the superoutburst is shown in figure 1. We also plot photometric data from the ASASSN CV Patrol (Shappee et al. 2014; Kochanek et al. 2017; Davis et al. 2015) that constrains the onset of the superoutburst. The superoutburst lasted about 21 days during BJD 2458452-2458473. The brightness first rapidly increased during BJD 2458448-2458452 and next slightly increased during BJD 2458452-2458462, reaching maximum at 15.2 
Table 1. Summary of OISTER observations.

\begin{tabular}{|c|c|c|c|c|c|}
\hline Telescope (Instrument) & Date (UT) & Exposure (Sec) & \multicolumn{3}{|c|}{ Filter (Number of data) } \\
\hline \multirow[t]{6}{*}{ SaCRA (MuSaSHI) $0.55 \mathrm{~m}$} & $2018-12-10$ & 30 & \multicolumn{3}{|c|}{ r $(810)$, i $(810)$, z $(810)$} \\
\hline & 2018-12-14 & 60 & \multicolumn{3}{|c|}{ r (206), i (206), z (206) } \\
\hline & 2018-12-17 & 60 & \multicolumn{3}{|c|}{ r (258), i (258), z (258) } \\
\hline & 2018-12-19 & 60 & \multicolumn{3}{|c|}{ r (488), i (488), z (488) } \\
\hline & 2018-12-23 & 60 & \multicolumn{3}{|c|}{ r (248), i (248), z (248) } \\
\hline & 2019-01-07 & 60 & \multicolumn{3}{|c|}{$\mathrm{r}(115), \mathrm{i}(115), \mathrm{z}(115)$} \\
\hline Kiso (Tomo-e Gozen) $1.05 \mathrm{~m}$ & 2018-12-10 & 7.5 & \multicolumn{3}{|c|}{ No filter (1181) } \\
\hline \multirow[t]{5}{*}{ MITSuME Akeno (Tricolor camera) $0.5 \mathrm{~m}$} & $2018-12-10$ & 60 & \multicolumn{3}{|c|}{$\mathrm{g}(79), \operatorname{Rc}(79), \mathrm{Ic}(79)$} \\
\hline & $2018-12-12$ & 60 & \multicolumn{3}{|c|}{$\mathrm{g}(105), \mathrm{Rc}(105), \mathrm{Ic}(105)$} \\
\hline & 2018-12-13 & 60 & \multicolumn{3}{|c|}{ g (25), Rc (25), Ic (25) } \\
\hline & 2018-12-14 & 60 & \multicolumn{3}{|c|}{ g (106), Rc (106), Ic (106) } \\
\hline & 2018-12-15 & 60 & \multicolumn{3}{|c|}{ g (106), Rc (106), Ic (106) } \\
\hline $\begin{array}{l}\text { MITSuME Okayama (Tricolor camera) } 0.5 \\
\text { m }\end{array}$ & 2018-12-08 & 60 & \multicolumn{3}{|c|}{$\mathrm{g}(52), \mathrm{Rc}(52), \mathrm{Ic}(52)$} \\
\hline \multirow[t]{12}{*}{ Kanata (HOWPol) 1.5 m } & $2018-12-10$ & 40 & \multicolumn{3}{|c|}{$\mathrm{I}(318)$} \\
\hline & 2018-12-13 & 40 & \multicolumn{3}{|c|}{$\mathrm{I}(74)$} \\
\hline & $2018-12-15$ & 40 & \multicolumn{3}{|c|}{ I (141) } \\
\hline & 2018-12-18 & 40 & \multicolumn{3}{|c|}{ I (108) } \\
\hline & 2018-12-19 & 40 & \multicolumn{3}{|c|}{ I (74) } \\
\hline & 2018-12-27 & 60 & \multicolumn{3}{|c|}{$\mathrm{V}(82), \mathrm{I}(69)$} \\
\hline & 2019-01-07 & 60 & \multicolumn{3}{|c|}{$\mathrm{V}(42), \mathrm{I}(53)$} \\
\hline & 2019-01-13 & 60 & \multicolumn{3}{|c|}{$\mathrm{V}(65), \mathrm{I}(66)$} \\
\hline & 2019-01-14 & 60 & \multicolumn{3}{|c|}{$\mathrm{V}(37), \mathrm{I}(75)$} \\
\hline & 2019-01-15 & 60 & \multicolumn{3}{|c|}{$\mathrm{V}(32), \mathrm{I}(41)$} \\
\hline & 2019-01-16 & 60 & \multicolumn{3}{|c|}{$\mathrm{V}(27), \mathrm{I}(41)$} \\
\hline & 2019-02-02 & 60 & \multicolumn{3}{|c|}{$\mathrm{V}(62), \mathrm{I}(61)$} \\
\hline \multirow[t]{2}{*}{ Murikabushi (Tricolor camera) $1.05 \mathrm{~m}$} & 2018-12-20 & 60 & $\mathrm{~g}(2$ & 8), Rc (218) & Ic (218) \\
\hline & 2018-12-24 & 60 & $\mathrm{~g}$ & 97), Rc (97), & c (97) \\
\hline Telescope (Instrument) & Date (UT) & Exposure (Sec) & Cover range & Resolution & Number of data \\
\hline Nayuta (MALLS) $2.0 \mathrm{~m}$ & 2018-12-10 & 1200 & $5130-7970$ & 1800 & 16 \\
\hline & 2018-12-24 & 1200 & $4490-9650$ & 800 & 3 \\
\hline & 2019-01-05 & 1200 & $4390-9550$ & 800 & 3 \\
\hline
\end{tabular}

Table 2. Journal of MDM Time-Series Photometry

\begin{tabular}{ccccc}
\hline \hline Start (UT) & HA range & Exp. (Sec) & $N$ & Filter \\
\hline 2019-09-05 07:38 & $-01: 36-+02: 41$ & 30 & 510 & GG420 \\
2019-09-06 07:11 & $-01: 59-+02: 15$ & 60 & 249 & R \\
2019-09-08 06:15 & $-02: 48-+01: 40$ & 30 & 357 & GG420 \\
2019-10-22 03:37 & $-02: 30-+02: 43$ & 30 & 553 & GG420 \\
2019-10-23 07:18 & $+01: 16-+03: 42$ & 30 & 274 & GG420 \\
2019-10-24 03:03 & $-02: 56-+02: 11$ & 33 & 475 & GG420 \\
\hline \hline
\end{tabular}


mag, and then began to decrease. The main superoutburst ended at BJD 2458473. There were two rebrightenings (during BJD 2458474-2458477 and 2458483-2458486) after the main superoutburst. After the rebrightenings, the magnitude returned to near the quiescent state around 17.5 mag. The superoutburst amplitude was $\sim 2.3$ mag.

\subsection{Orbital period}

For analysis, we first subtracted the global trend of the light curve of the superoutburst and rebrightenings by subtracting a smoothed light curve obtained by locally weighted polynomial regression (LOWESS: Cleveland 1979) After the subtraction, we performed a phase dispersion minimization (herafter PDM, Stellingwerf 1978) analysis of whole superoutburst and found the orbital period to be $P_{\text {orb }}=0.149454(3) \mathrm{d}$. We then derived the phaseaveraged profile of the eclipse folded on the orbital period. To determine the mid-eclipse time, we fitted the Gaussian profile and derived BJD 2458461.428617 as the epoch of the eclipse.

\subsection{Superhump period}

We removed all eclipses to investigate the variation of the superhump period during the superoutburst. We calculated the time of eclipses from the above epoch and orbital period and masked eclipses with a range of 0.11 in units of orbital phase.

Figure 2 shows the $O-C$ curve (upper panel), the amplitude of the superhumps (middle panel), and the light curve (lower panel) of ASASSN-18aan during BJD 2458458-58474. We determined the times of maxima of superhumps in the same way as in Kato et al. (2009). The resulting times are listed in table E3.

From the variation of the superhump period and the amplitudes of superhumps, we regarded BJD 2458455.0$2458462.5(0 \leq E \leq 22)$ as stage A, BJD 2458462.5$2458467.6(29 \leq E \leq 56)$ as stage B, and BJD 2458467.6$2458473.5(60 \leq E \leq 93)$ as stage $\mathrm{C}$ superhumps.

The PDM analysis of stage A superhumps (upper panel) and the phase-averaged mean profile (lower panel) are shown in figure 3. Single-peaked, asymmetric modulations are evident, with an amplitude of $0.3 \mathrm{mag}$. We found the stage A superhump period to be $P_{\mathrm{stA}}=0.16282(5) \mathrm{d}$. We also found the stage $\mathrm{B}$ and stage $\mathrm{C}$ superhump period to be $0.15821(4) \mathrm{d}$ and $0.14944(5) \mathrm{d}$, respectively. Note that stage $\mathrm{C}$ superhump period is equal to the orbital period within the error. This indicates that ellipsoidal variations by the secondary contaminated superhumps in stage C. To derive a plausible period of stage $\mathrm{C}$ superhumps, we subtracted the phase-averaged profile of quiescent light curve in BJD 2458490-2458520, which shows apparent ellipsoidal variations. After the subtraction, we performed PDM analysis again and derived the period of $0.15743(6)$ d. This period is shorter than that of stage B by $0.5 \%$ and consistent with ordinary cases (Kato et al. 2009); therefore, we adopt this as the stage $\mathrm{C}$ superhump period.

$P_{\text {dot }}\left(\equiv \dot{P}_{\mathrm{sh}} / P_{\mathrm{sh}}\right)$, which is the derivative of the superhump period during stage $B$, is $91(34) \times 10^{-5}$. This value is quite large, but less reliable because of the large error and uncertainty of the boundary of each superhump stage stemmed from small number of points.

\subsection{Variation of phase-averaged profile through superoutburst}

Figure 4 shows the phase-averaged profile folded on the orbital period. We divided the light curve of the superoutburst into three stages; superoutburst stage (BJD 2458453-2458473.5) including a whole main superoutburst, rebrightening stage (BJD 2458473.5-2458487) including two rebrightenings, and quiescent stage (BJD 24584872458520) which is after the rebrightenings. It is evident that the eclipse clearly seen at the superoutburst stage around phase 0.0 becomes shallow at the rebrightening stage, and then it can be hardly seen at the quiescent stage. This indicates the inclination $i$ of this system is small for an eclipsing system $\left(i \sim 80^{\circ}\right)$, and the eclipses at the superoutburst and rebrightening stages are caused by the secondary star passing the front of the expanded, bright disk. It is clearly seen that the light curve at the superoutburst or rebrightening stage is affected by ellipsoidal variations. We discussed the detail of these ellipsoidal variations based on the MDM observation data as below.

\subsection{Variation of spectral characteristics through superoutburst}

Figure 5 shows spectra through the superoutburst. The spectra were obtained during the brightening state, just after the first rebrightening, and in the quiescent state after the second rebrightening. As described in section 2.3, although a sensitivity correction was not carried out in all observations the profile transition can be seen by comparing each trace. The disk component rose at shorter wavelength during the superoutburst, while it became weak at the quiescent state. In addition to this, the $\mathrm{H} \alpha \lambda 6563$ line went from emission in outburst to absorption in quiescence, indicating an appearance of the secondary component. 


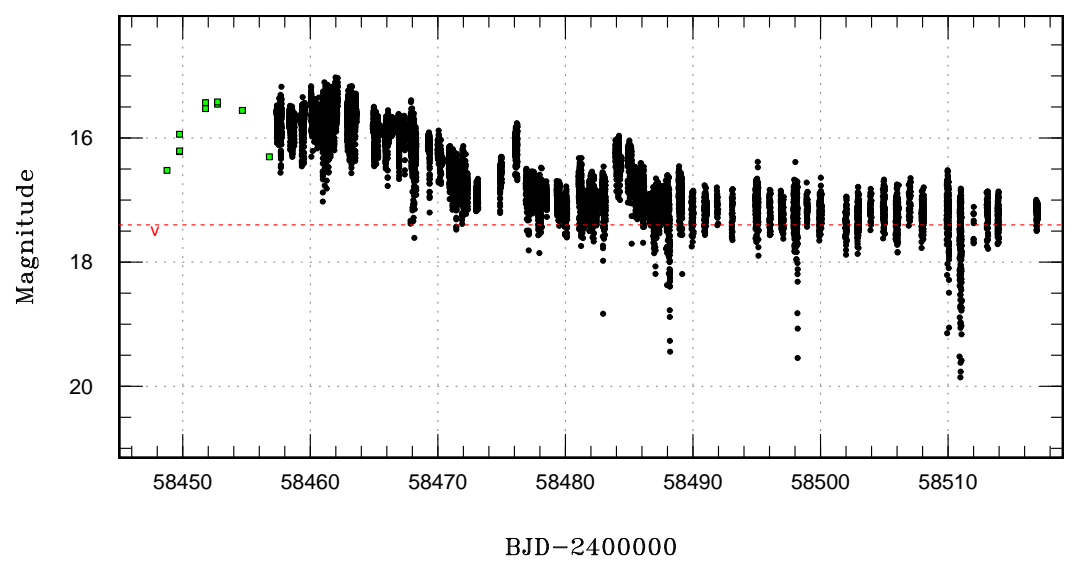

Fig. 1. Light curve of ASASSN-18aan. The filled- squared points show the ASAS-SN's V magnitude. The V-shaped sign represents an upper limit by ASAS$S N$ 's $V$ and $g$ magnitude, and the quiescent magnitude is shown as a red dashed line.

Cycle (E)

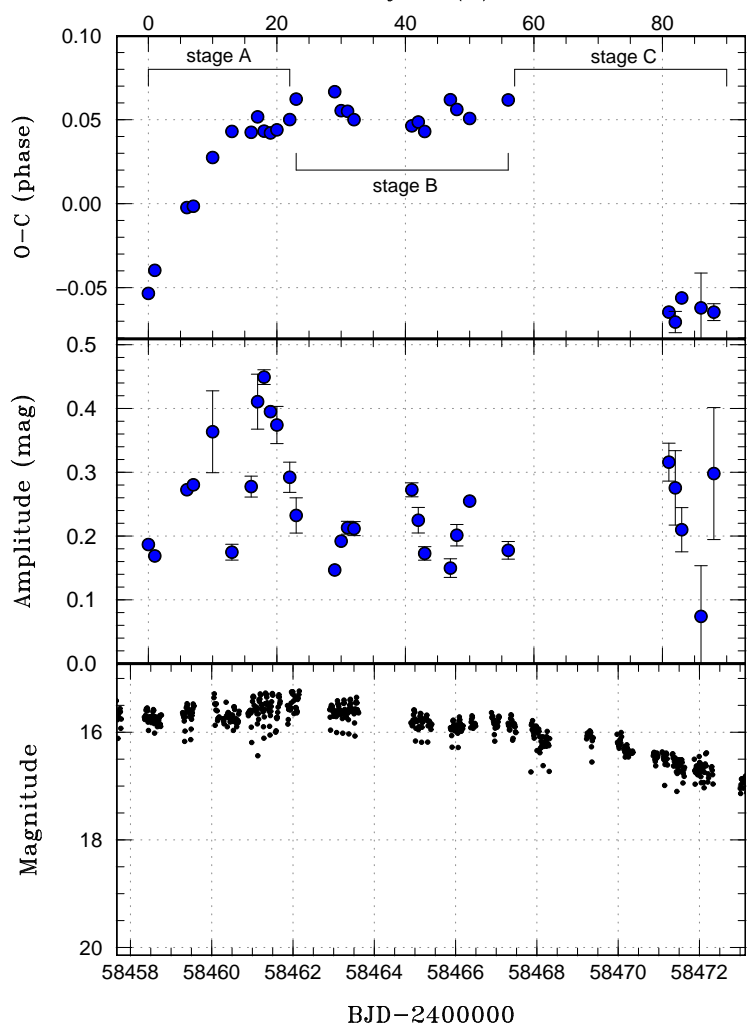

Fig. 2. Upper panel: $O-C$ curve of ASASSN-18aan during BJD 245845858472. We used an ephemeris of BJD 2458461.485+0.1579E for drawing this figure. Middle panel: Amplitude of superhumps. Lower panel: Light curve. The horizontal axis in units of BJD and cycle number is common to all of these panels.
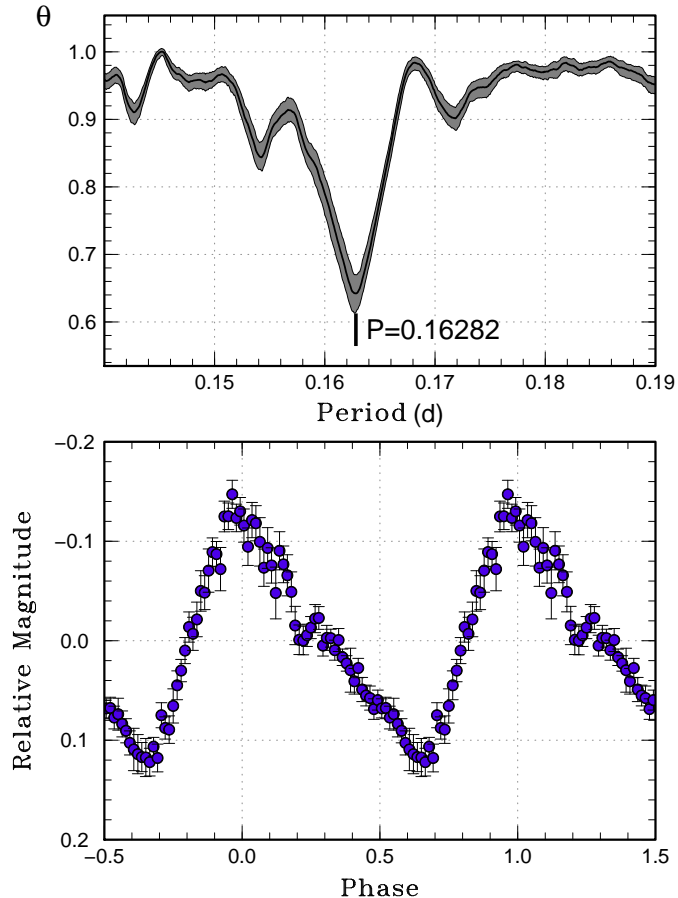

Fig. 3. Upper panel: $\theta$-diagram of our PDM analysis of stage A superhumps of ASASSN-18aan (BJD 2458455.0-2458462.5). The gray area represents the $1 \sigma$ error of the best-estimated period by the PDM method. Lower panel: Phase-averaged profile of stage A superhumps. 


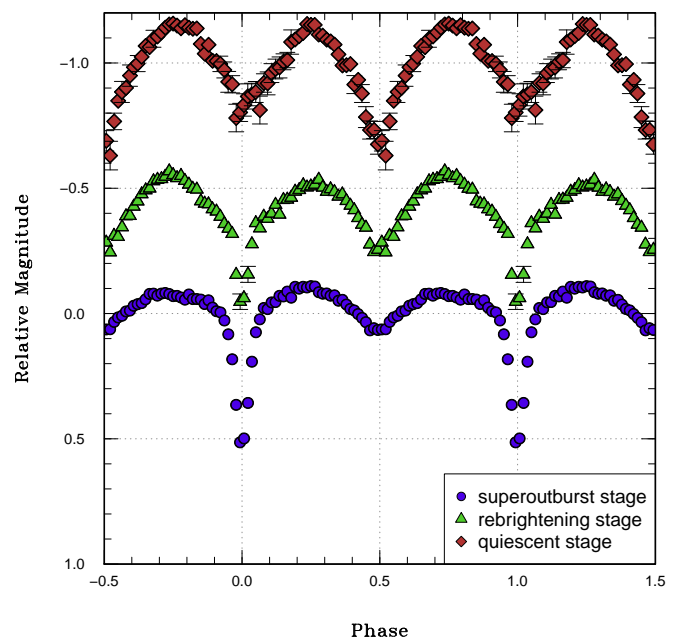

Fig. 4. Phase-averaged profiles of ASASSN-18aan during the superoutburst stage (BJD 2458453-2458473.5; blue circles), rebrightening stage (BJD 2458473.5-2458487; green triangles) and quiescent stage (BJD 24584872458520; red diamonds).

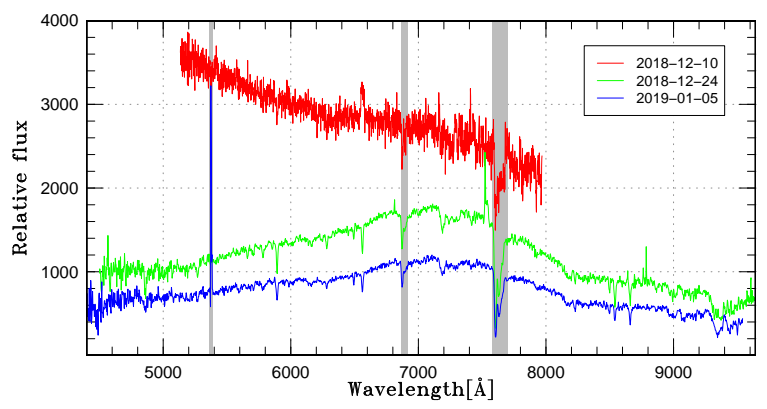

Fig. 5. Average flux. The gray area represents a contamination of the atmosphere or noise. The spectra shown are from 2018-12-10, 2018-12-24 and 2019-01-05 (UT) from top to bottom, respectively. The trace for 2018$12-10$ is shifted above by 1600 units for convenience. The spectra are not flux-calibrated (see section 2.3).

\subsection{Photometric observations in quiescent state}

Figure 6 shows MDM light curves from 2019 September and October, after the source had returned to quiesence. The light curves show a persistent modulation with two peaks per orbit. This ellipsoidal variation indicates a strong contribution from a tidally-distorted secondary star. Such strong ellipsoidal variation is seldom observed in CVs with periods this short.

Several features of the quiescent light curve are not consistent with a pure ellipsoidal variation. Most obviously, shallow eclipses appear around phase zero. The mean magnitude is about $0.1 \mathrm{mag}$ fainter in 2019 October than in the previous month, while the secondary star should be nearly constant; evidently the disk faded over the interval

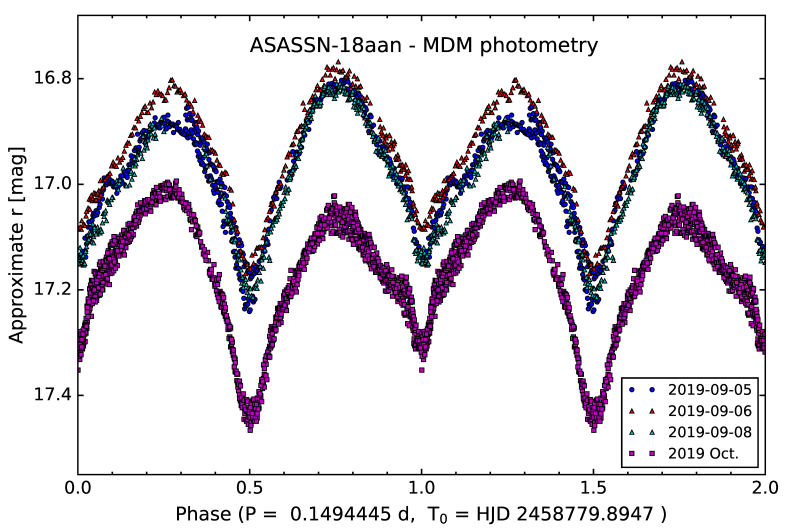

Fig. 6. Light curves from the MDM $1.3 \mathrm{~m}$ telescope, folded on the eclipse ephemeris, and repeated for a second cycle for continuity.

between observations. In the September data, the peaks near phase 0.75 are slightly brighter than those near 0.25 , while in the October data the opposite is true; in pure ellipsoidal variation they should be exactly equal. Also, there are subtle but clear breaks in the slope near phases 0.4 and 0.6. These are clearest in the October data, where the mean light level is a bit fainter. The interval around phase 0.5 was covered on all three nights in the October data, so this feature is highly reproducible. We consider its interpretation later.

Figure 7 gives a magnified view of the eclipse in the quiescent light curves. The eclipse is only $\sim 0.1 \mathrm{mag}$ deep, but it is clearly detected in all the data sets, with ingress starting around phase 0.96 and last contact around phase 0.04 . The eclipse morphology is reminiscent of the longer-period CV ASASSN-15aa (see Figs. 12 and 13 of Thorstensen et al. 2016), which also shows a shallow eclipse superposed on a strong ellipsoidal variation.

\subsection{Spectroscopy and velocities}

The mean spectrum (figure 8) shows weak emission, but also absorption features characteristic of a late-type star, as might be expected given the strong ellipsoidal modulation.

To explore how the spectrum behaves through the orbital cycle, we prepared a phase-averaged representation of our spectral data. To start, we rectified each spectrum by dividing by its fitted continuum and computed the phase of each spectrum using the photometric ephemeris. We then constructed a grid of equally-spaced phases, and at each phase computed an average of the phase-adjacent rectified spectra, weighted by a narrow Gaussian centered on 


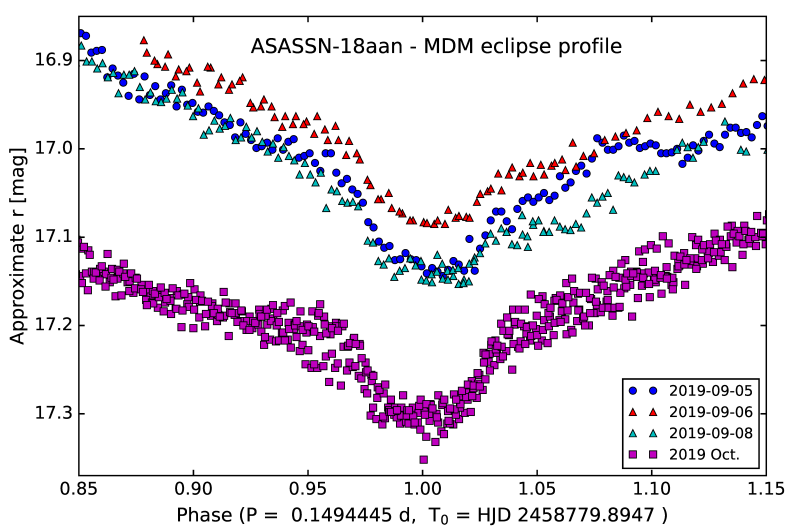

Fig. 7. Magnified light curves of the primary eclipses around phase 1.0. Other conditions are same as figure 6.

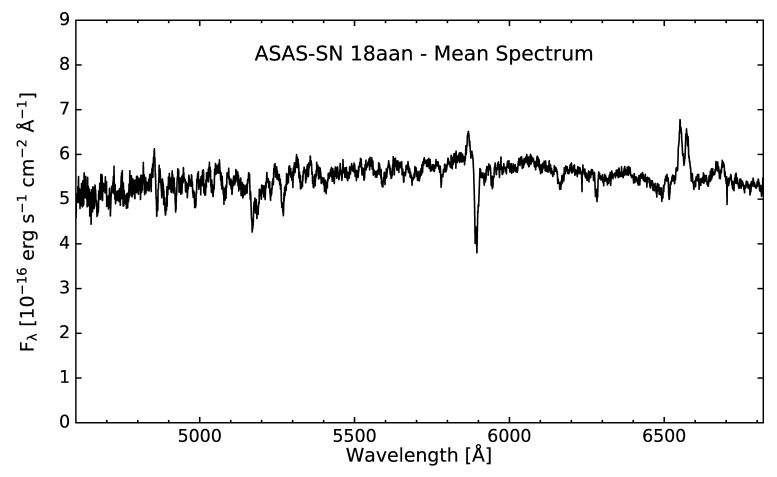

Fig. 8. Average flux-calibrated spectrum from 2019 Sept.

the fiducial phase. Figure 9 shows a portion of the twodimensional image formed by stacking these averages. The absorption lines vary strikingly in velocity on the orbital period, conclusively demonstrating that they are from the secondary star rather than some other object. Emission is visible at $\mathrm{H} \alpha \lambda 6563$, but its profile is complicated by the secondary's $\mathrm{H} \alpha$ absorption line, which reveals itself through its velocity modulation.

Note that the emission lines at $\mathrm{H} \alpha$ and $\mathrm{HeI} \lambda \lambda 5876,6678$ all show transient absorption features near phase 0.5. We will return to this later.

We measured the radial velocities of the secondary component by cross-correlating the observed spectrum with a template spectrum using the IRAF task fxcor; Tonry, Davis (1979) describe the principle. The template was a composite of 76 spectra of later-type IAU velocity standards that had been shifted to zero apparent radial veloc-

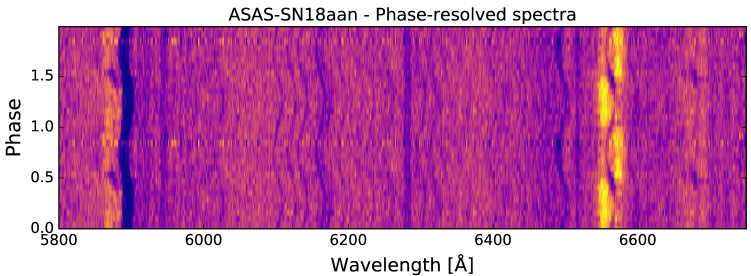

Fig. 9. Phase-resolved spectrum. Note the radial velocity variation of the absorption lines.

ity before averaging. The correlations excluded the region near the sodium D lines because of possible confusion with HeI $\lambda 5876$ emission. All our spectra gave good correlations despite the exclusion of this strong feature. We corrected our velocities $v$ to the reference frame of the solar system barycenter and fitted them with a sinusoid of the form

$v(t)=\gamma+K \sin \left[\frac{2 \pi\left(t-T_{0}\right)}{P}\right]$,

where $t$ is the barycentric time of mid-integration. We fixed $P$ to the eclipse period, and found

$$
\begin{aligned}
\gamma & =7 \pm 4 \mathrm{~km} \mathrm{~s}^{-1} \\
K & =273 \pm 6 \mathrm{~km} \mathrm{~s}^{-1}, \\
T_{0} & =\text { BJD } 2458732.9708 \pm 0.0005, \text { and } \\
\sigma & =13 \mathrm{~km} \mathrm{~s}^{-1},
\end{aligned}
$$

where the time base is UTC and $\sigma$ is the standard deviation around the best fit. Allowing $P$ to float yielded $P=0.1486(9) \mathrm{d}$, consistent with the much more accurate eclipse-based period, and had almost no effect on the values of the other parameters. Figure 10 shows the radial velocities and the fit.

To study the secondary star's spectrum, we averaged our fluxed spectra, after shifting them to the rest frame of the secondary star using the parameters above. We de-reddened the spectrum using $E(B-V)=0.36$ (see the Introduction). We then used an interactive program to subtract spectra of different (known) spectral types and normalizations from this rest-frame average, with the aim of cancelling the stellar absorption features and leaving the smooth continuum characteristic of accretion-disk spectra between the emission lines. Some of the template spectra we used were from our own observations of stars classified by Keenan, McNeil (1989), and others were originally published by Jacoby et al. (1984). Figure 11 shows the best result, in which the subtracted spectrum is of the G9V star HD29050 (from Jacoby et al. 1984) scaled to an unreddened flux equivalent to $V=16.3$, which would be $V=17.5$ after reddening. 


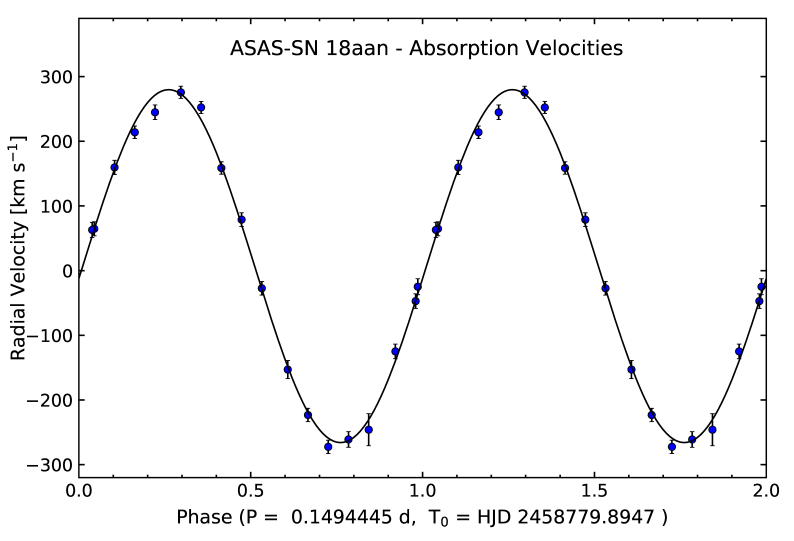

Fig. 10. Absorption-line velocities folded on the orbital ephemeris, with the best-fit sinusoid superposed. All points are repeated over a second cycle for continuity.

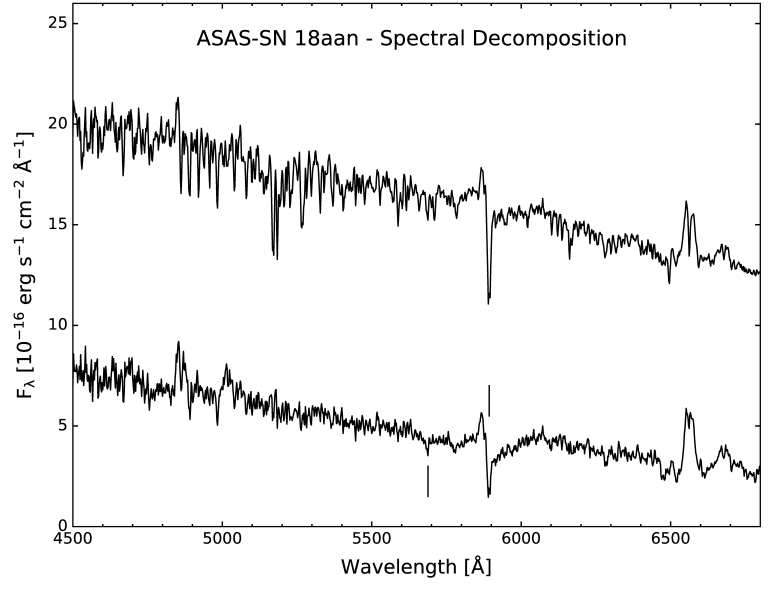

Fig. 11. (Upper trace): Rest-frame average spectrum, dereddened by 1.19 magnitudes. (Lower trace): The same, after subtraction of a scaled spectrum of a G9V-type star. The solid vertical lines mark the wavelengths of sodium features near $\lambda 5690$ and the very strong $\mathrm{NaD}$ doublet near $\lambda 5893$.

\section{Analysis}

\subsection{System parameters from superhumps and eclipse modeling}

We estimated the mass ratio of ASASSN-18aan in the same way as proposed in Kato, Osaki (2013) by using the fractional superhump-period excess for the 3:1 resonance, $\varepsilon *=1-P_{\text {orb }} / P_{\text {stA }}$. We calculated $\varepsilon *=0.0821(3)$ using the orbital period determined from the eclipses and the stage A superhump period. We estimated the mass ratio of ASASSN-18aan to be $q=0.278(1)$ by using equation (1) and (3) in Kato, Osaki (2013).

We studied eclipses during the superoutburst. We performed intensive, multicolor time-series photometric observations especially at the timing of eclipses via VSNET and OISTER

Before analysis of eclipses, we subtracted the global trend of the light curve in the same way as section 3.1. We next subtracted the influence of the primary and the bright secondary, which causes ellipsoidal variations in quiescent state. We simply subtracted the phase-averaged profile in quiescence, BJD 2458490-2458520, as described in section 3.3. This is because there seems to be no eclipse of the accretion disk in the quiescent state, judged from figure 4 or 7 . We see no indication of accretion-disk features such as the hot spot, and thus the light source of quiescent state is considered to be only components of the primary and the secondary. After the subtraction of the contribution of ellipsoidal variations, we also subtracted the variation of superhumps. We used a same way in principle as the case of subtraction of ellipsoidal variations. The superhump profile, however, varies from moment to moment (see, e.g., figure 8 in Patterson et al. (1995)). We therefore divided the superoutburst into short intervals and computed phase-averaged superhump profiles in each interval. We finally removed them from the light curve by dividing the light curve by them.

The modeling of the eclipsing light curve was constructed mostly in the same way as Kato et al. (2014a) and Kato et al. (2015). We assumed that radial dependence of the disk surface brightness follows $r^{p}$, where $r$ is the disk radius and $p$ is the exponent. Under the assumption of the accretion disk to be the black body and steady in time, $p=-3 / 4$. Since we subtracted the component of the primary from the light curve in advance, we assumed the disk has an inner rim. By using the white dwarf mass estimated in section 4.2 and 4.3 , we estimated the white dwarf radius (equal to the inner rim of the disk) to be $R_{\text {inner }}=0.011 a$, where $a$ is a binary separation (Nauenberg 1972). The secondary fills its Roche lobe.

We set the disk radius $r$, exponent $p$ and inclination $i$ 
as free parameters and estimated them by Markov Chain Monte Carlo (MCMC) method. We use the mass ratio, $q=0.278(1)$, estimated from the stage A superhump because the relation between the mass ratio and inclination is quite strong that it takes much more time to converge. See Appendix for the detail of the settings of this method.

We first tried to estimate the inclination $i$. At the maxima or minima of superhumps it is difficult to make better phase-averaged profile from a few neighboring, continuously changing superhumps, leading to incorrect estimation. We thus selected three excellent eclipses which exist on the hillside of superhumps and have almost linear wings, using eclipse $9 \mathrm{a}, 9 \mathrm{~b}$ and 52 in table E1. We estimated the disk radius, exponent and inclination simultaneously, and derived the value of inclination to be $76.54_{-0.05}^{+0.06 \circ}, 76.70_{-0.03}^{+0.03 \circ}, 76.56_{-0.04}^{+0.04 \circ}$ (errors are calculated from the $95 \%$ credible intervals), and set the inclination to be $76.60(9)^{\circ}$. Hereafter, in this section we use this value as the inclination and fixed in our estimation.

Figure 12 shows the result of our estimation. We analyzed 48 eclipses through the superoutburst and two rebrightenings. We did not investigated the eclipse in quiescence because of its shallow depth and low signal-to-noise ratio. We additionally note that during the rebrightenings we obtained limited-quality data on only five eclipses.

The effective radius of the Roche lobe (Eggleton 1983), the tidal truncation radius $R_{\text {tidal }} / a=0.60 /(1+q)$ (approximated equation derived from Paczyński (1977)), the 3:1 resonance radius and the circularization radius (Hessman, Hopp 1990) are indicated in Figure 12. The disk radius seems to shrink through the superoutburst. Here note that the disk radius mainly corresponds to the width of the ingress and egress of the eclipse, and thus the effect of subtracting the ellipsoidal variations and superhumps is rather small, implying the estimated values are fairly reliable. On BJD 2458457, 5 days after the onset of the superoutburst inferred from the ASAS-SN observation, the disk radius is around $0.4 a$, decreasing almost linearly and then reaching around $0.2 a$. The radius at the first and second rebrightening is near the circularization radius. A tendency with difference of color are clearly seen; eclipses observed in the B-band filter correspond to small radii, those in Ic or Rc-band are wider corresponding to large radii, and ones by $\mathrm{C}$ or $\mathrm{V}$-band filter are intermediate between these two. This results are good agreement with the basic picture of the accretion disk, which should be hottest in the inner part and cooler at larger radii.

The disk radius is largest at the onset of the superoutburst, and comparable to the $3: 1$ resonance radius. To investigate this, we performed a linear regression analysis to the data only including eclipses by $\mathrm{C}$ and $\mathrm{V}$-band fil-

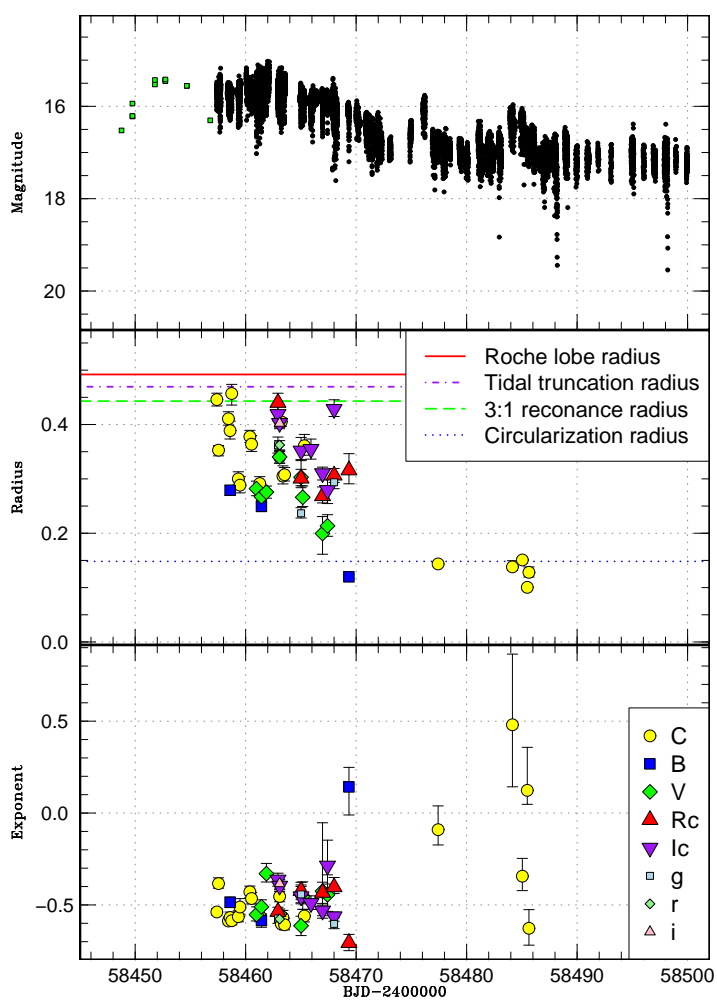

Fig. 12. Upper panel: Light curve. The filled-square points show the ASASSN's V magnitude. Middle panels: Radius in units of binary separation. Bottom panel: Exponent. All error bars are calculated from $95 \%$ confidence intervals of the posterior probability. The horizontal axis in units of BJD is common to all of these panels. The points labeled "Ic" include data from both the I and Ic passbands.

ter in main superoutburst, and estimated the disk radius at the onset of the superoutburst to be $0.47 a$. This value is larger than the 3:1 resonance radius, $0.44 a$, indicating that the superoutburst of ASASSN-18aan may be caused by indeed the tidal instability.

The power-law brightness exponent $p$ strongly affects the eclipse depth. Our estimated values seem to be concentrated around -0.5 , being small compared with the value assuming the steady-state black body, -0.75 . This might be an artifact of subtracting the ellipsoidal variations and superhumps, which may artificially reduce the eclipse depth. In addition, the MDM observations show shallow eclipses in quiescence at the phase 0.96-1.04 (set phase 1.0 to the mid eclipse). However, the primary eclipse with the radius of $R_{\mathrm{WD}} / a=0.011$ occurs at the phase 0.98 01.02 , indicating the eclipses in quiescence include somewhat disk components. Our estimated values therefore might be systematically small because of oversubtraction. 


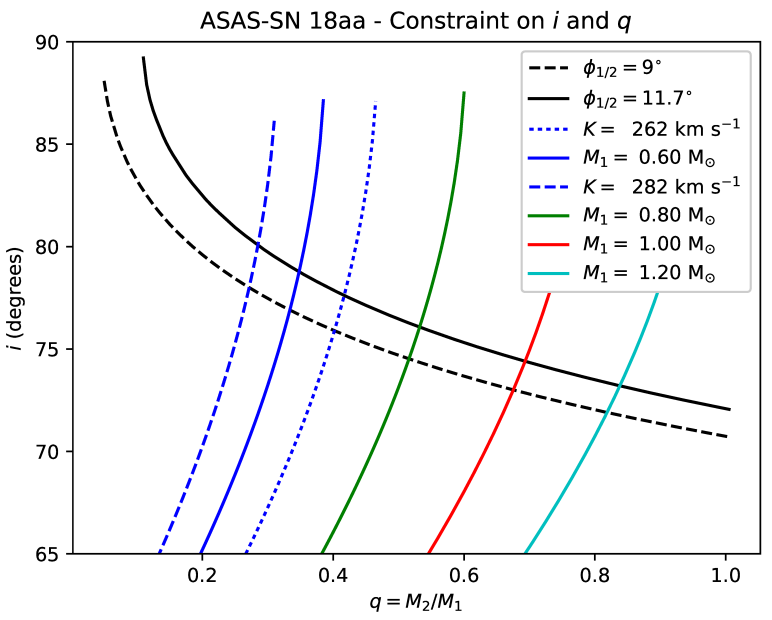

Fig. 13. Constraints from $K_{2}$ (vertical curves) and the eclipse width (downward-sweeping curves) in the $i-q$ plane.

\subsection{System parameters from velocity amplitude}

We further constrain the system parameters using our measured velocity amplitude for the secondary star, $K_{2}$. Figure 13 shows the constraints in $i-q$ plane. The solid, roughly vertical curves are for various assumed white dwarf masses, $M_{\mathrm{WD}}$, for our best fit value of $K_{2}$. The dashed and dotted lines flanking the leftmost (blue) curve illustrate the effect of a generous $10 \mathrm{~km} \mathrm{~s}^{-1}$ uncertainty in $K_{2}$. The curves sweeping down from the upper left are the constraints for different eclipse half-widths, $\Delta \phi_{1 / 2}$. The constraint on $q$ implies $M_{\mathrm{WD}} \sim 0.6 \mathrm{M}_{\odot}$, so that $M_{2}=q M_{\mathrm{WD}} \sim 0.18 \mathrm{M}_{\odot}$. The white dwarf mass is similar to average field white dwarfs, and on the low side compared to most CVs, for which Zorotovic et al. (2011) give an average $M_{\mathrm{WD}}=0.83 \pm 0.23 \mathrm{M}_{\odot}$, where the standard deviation is for the distribution rather than the standard deviation of the mean. The secondary mass is much less than that of a main-sequence G9 star; a main-sequence star of such low mass would have a spectral type around M4 or M5 (Pecaut, Mamajek 2013).

\subsection{Modeling ellipsoidal variations}

We built a model of the ellipsoidal variations using a code developed by Thorstensen, Armstrong (2005), which models only the contribution from the Roche-lobe-filling secondary star. The code computes the Roche lobe's physical size and shape for assumed primary and secondary masses, and the surface brightness of each element as viewed from earth including gravity and limb darkening, using empirical surface brightnesses appropriate to the spectral band and surface temperature. After computing the brightness at each phase, the code adjusts the normalization by finding the distance for which the predicted light curve matches the data. We used the 2019 October MDM light curve, which has the smallest disk contribution and excellent night-to-night reproducibility, scaled the broad-band points to approximate $V$ magnitudes as described above, and subtracted $1.19 \mathrm{mag}$ to correct for extinction. Points that appeared to be affected by the primary or secondary eclipses were masked. We set the secondary's $T_{\text {eff }}$ to 5300 kelvin, appropriate for its G9 type (Pecaut, Mamajek 2013), as well as $M_{\mathrm{WD}}=0.6$ and $M_{2}=0.18 \mathrm{M}_{\odot}$ and $i=77^{\circ}$. To the light curve we added a non-variable cwomponent equivalent to $F_{\lambda}=5 \times 10^{-16} \mathrm{erg} \mathrm{cm}^{-2} \mathrm{~s}^{-1} \AA^{-1}$, about the same as the disk contribution shown in Figure 11,

Figure 14 shows the result. We found we could not match the deep minima around phase 0.5 without a secondary eclipse. Also, without the extra light from the disk, the predicted amplitude of the ellipsoidal variation at the system's inclination was too great. We have one other bit of evidence that supports the secondary-eclipse interpretation: referring back to figure 9 , we notice transient $a b$ sorption reversals near phase 0.5 in He I $\lambda \lambda 5876$ and 6678 and also enhanced $\mathrm{H} \alpha$ absorption. This strongly suggests that the secondary's light is passing through a substantial column of high-excitation gas at those phases, as might be expected in the atmosphere of the disk.

The distance obtained by scaling the ellipsoidal variation model to the observed light curve is $624 \mathrm{pc}$, which, in view of the long chain of reasoning that went into the choice of parameters, agrees well with the Gaia DR2 parallax determination $(675+32,-29 \mathrm{pc})$. The overall picture is remarkably self-consistent $-M_{\mathrm{WD}} \sim 0.6$ and $M_{2} \sim 0.18 M_{\odot}$, a secondary with $T_{\text {eff }}$ above $\sim 5000$ kelvin, and $i \sim 77^{\circ}$.

\section{Discussion}

\section{$5.13: 1$ resonance condition in long-period objects}

As mentioned in section 4.1 , the disk radius at the onset of the superoutburst seems to reach the 3:1 resonance radius. This indicates the superoutburst of ASASSN-18aan is indeed caused by the tidal instability in the accretion disk, and thus the upper limit of critical mass ratios of the $3: 1$ resonance would be larger than $q=0.278$. This result presents one possibility that superoutbursts of other long- $P_{\text {orb }}$ objects also may be caused by the tidal instability at the 3:1 resonance radius. We therefore investigated SU UMa-type DNe that have long orbital periods and the mass ratios are (even partially) estimated. We summarized the results in table 3 . There are some objects beyond the 


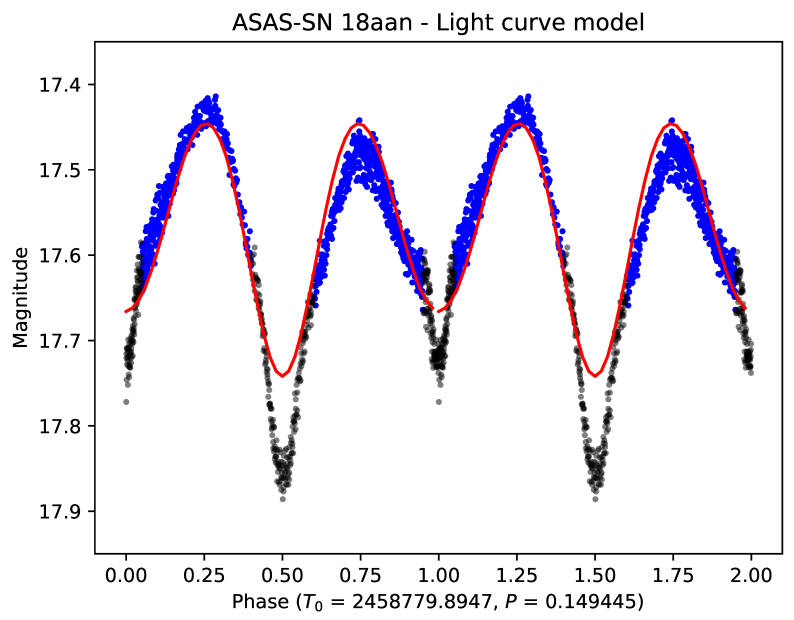

Fig. 14. Light curve from 2019 October. The red curve shows the model of the ellipsoidal variation described in the text. The larger blue points were used in the normalization, and the smaller black points near phase 0.5 and phase 0 were ignored. The modeled light curve has been extinguished by $A_{V}=1.19$ mag to match the observed points.

upper limit of the tidal instability. Most of them, however, are less reliable values. First of all, in almost half of the listed objects, mass ratios are estimated with specific ranges. Objects except TU Men and NY Ser have lower mass ratio than the upper limit of 0.25 (Whitehurst 1988 ) or 0.33 with reduction of mass transfer (Murray et al. 2000).

Kato et al. (2019b) estimated a referential value of mass ratio of NY Ser from the superhump period of the postsuperoutburst stage as proposed by Kato, Osaki (2013). In this method, the mass ratio is calculated by assuming ignorance of the pressure effect in the accretion disk, which conditions are considered to be fulfilled during the stage A and post-superoutburst stage, and using the orbital period, superhump period and disk radius. The range of the estimated mass ratio of NY Ser arose from the supposed disk radius. The disk radius at the post-superoutburst, however, is uncertain (Kato, Osaki 2013), and thus the mass ratio is quite indistinct.

TU Men has seriously large mass ratios for the tidal instability. Stolz, Schoembs (1984) derived the mass ratio to be $q=0.59$ from spectroscopic observations during the 1980 superoutburst. Mennickent (1995) estimated the mass ratio to be $q=0.455(45)$ from $\mathrm{H} \alpha$ emission line in quiescent state. He also reported second candidate of its mass ratio to be $q=0.33$. Smak (2006) reanalyzed the radial velocity measured by Mennickent (1995) and calculated the mass ratio to be $q=0.5(2)$, or the lower limit to be $q>0.41(8)$ independently. The mechanism of superout- burst in TU Men is yet unclear. More precise observations should be needed to conclude whether the tidal instability acts on the accretion disk in TU Men.

We should note in the case of U Gem. This object showed long outburst in 1985, and Smak, Waagen (2004) reported detection of superhump signal in this outburst. The mass ratio of U Gem is 0.364(17) (Smak 2001), beyond the upper limit of the tidal instability in a disk. If U Gem truly showed superhumps in past outburst, other mechanism like enhanced mass transfer model should be needed. It is, however, proposed that this signal is doubtful (Schreiber 2007, Kato et al. 2014b), and there are much more rooms for further discussion before conclusion.

From above confirmation, we conclude that the tidal instability may act well in long- $P_{\text {orb }}$ SU UMa-type DNe, excepting TU Men. Kato et al. (2017) indicated that above the period gap, DNe on the standard CV evolutionary track hardly experience the 3:1 resonance; long- $P_{\text {orb }} \mathrm{SU}$ UMa-type DNe seem not to be on the standard track. As discussed in section 5.4, ASASSN-18aan has anomalously warm secondary, which evidently puts it on an unusual evolutionary track. This is also the case in ASASSN-14ho, another long- $P_{\text {orb }}$ SU UMa-type DNe having a warm secondary (Gasque et al. 2019). These results coincide with the argument by Kato et al. (2017).

\subsection{Tidal truncation radius in SU UMa-type and WZ Sge-type DNe}

Paczyński (1977) calculated the restricted three-body problem and derived the last non-intersecting orbit around the primary assuming an inviscid, pressure-free disk. This last non-intersecting orbit is considered to be the largest radius of a disk, known as the tidal truncation radius. Several authors have considered the tidal influence at the outer rim of the disk, and obtained nearly identical results (Ichikawa, Osaki 1994; Papaloizou, Pringle 1977; Truss 2007). There is, however, some room for discussion about the tidal truncation radius, especially in extremely low- $q$ systems. Lin, Papaloizou (1979) showed that at extremely small mass ratios, the outer rim is not truncated and extends beyond the primary Roche lobe. Hellier (2001) suggested the multiple rebrightenings called echo outbursts in ER UMa systems cause a weak angular momentum dissipation in the hot, eccentric disk due to the extremely low mass ratio. The cause of the discrepancy between the observation and theoretical predictions in WZ Sge-type $\mathrm{DNe}$ is unknown. The condition that the 3:1 resonance radius lies inside the tidal truncation radius is realized in the case of $q<0.25$, being consistent with observations, most of SU UMa-type DNe having lower than this value. 
Table 3. List of long $P_{\text {orb }}$ SU UMa-type DNe candidates which the mass ratios are estimated.

\begin{tabular}{|c|c|c|c|c|}
\hline Object & $P_{\mathrm{Porb}}{ }^{*}$ & 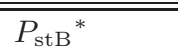 & $q$ & References \\
\hline$\overline{\text { SDSS J162520.29+120308.7 }}$ & $0.09113(30)$ & $0.09604(3)$ & $0.21(1)$ & 1 \\
\hline NY Ser & $0.09744(5)$ & 0.10458 & $0.23-0.43$ & 2,3 \\
\hline CzeV404 & $0.0980203(6)$ & $0.10472(2)^{\dagger}$ & 0.32 & 4 \\
\hline V1006 Cyg & $0.09832(15)$ & $0.10541(4)$ & $0.26-0.33$ & 5,6 \\
\hline MN Dra & $0.0998(2)$ & 0.105040 & $0.258(5)-0.327(5)$ & 7,8 \\
\hline TU Men & $0.1172(2)$ & $0.12558(4)^{\dagger}$ & $0.5(2)$ & 9,10 \\
\hline OT J002656.6+284933 (= CSS101212:002657+284933) & - & $0.13225(1)^{\dagger}$ & $0.10-0.15$ & 11 \\
\hline ASASSN-14ho & $0.24315(10)$ & - & 0.28 & 12 \\
\hline ASASSN-18aan & $0.149452(2)$ & $0.15790(5)$ & $0.278(1)$ & $\begin{array}{l}\text { This pa- } \\
\text { per. }\end{array}$ \\
\hline
\end{tabular}

*In units of day.

${ }^{\dagger}$ Averaged period.

1.Montgomery et al. (2017); 2. Kato et al. (2019b); 3. Pavlenko et al. (2014); 4. Ba̧kowska et al. (2014); 5. Kato et al. (2016)

6. Pavlenko et al. (2018); 7. Pavlenko et al. (2010); 8. Kato et al. (2014a); 9. Mennickent (1995); 10. Smak (2006)

11. Kato et al. (2017) ; 12 Gasque et al. (2019)

On the other hand, the condition that the 2:1 resonance radius lies inside the tidal truncation radius holds only if $q<0.025$. This is much lower than the value derived from observations, which concentrates around 0.06-0.08 (Kato 2015). Osaki, Meyer (2002) suggested that in extremely low mass-ratio system such as WZ Sgr-type DNe, the tidal torque at the tidal truncation radius may be too weak to stop the sudden expansion of the disk due to the outburst, and the outer rim of the disk would go beyond the tidal truncation radius and reach the 2:1 resonance radius. Wakamatsu et al. (2017) indicated that tidal dissipation at the tidal truncation radius in ASASSN-16eg, WZ Sge-type DNe having anomalously large mass ratio of $q=0.166(2)$, hardly worked. The $2: 1$ resonance radius of ASASSN-16eg lies outside the radius of the primary Roche lobe and, of course, the tidal truncation radius.

By combining our results with these, we indicate that the tidal torque at the tidal truncation radius indeed acts well in SU UMa-type DNe; however, it does not operates effectively in WZ Sge-type DNe. As discussed in section 5.1 , even in long- $P_{\text {orb }} \mathrm{SU}$ UMa-type DNe the mass ratios seem to be under or near the upper limit, and thus the $3: 1$ resonance radius likely lies in the tidal truncation radius. This means the tidal dissipation at the tidal truncation radius acts effectively and the expansion of the disk limited by this radius. However, when the mass ratio becomes small and the tidal torque by the secondary also weakens, the $2: 1$ resonance radius is the last position that the angular momentum of the disk is effectively extracted. Paczyński (1977) showed that the range of unstable orbits, which first appear around $q=0.22$, shrinks in extremely low- $q$ systems. This results may indicate that this unstable orbits are unlikely to work as the point of tidal limitation for extremely low- $q$ systems such as WZ Sge-type DNe. If so, it is a reasonable probability that the tidal truncation radius in such extreme systems is no longer a hard limit to terminate disk expansion.

\subsection{Similarity to WZ Sge-type dwarf novae}

We regarded the duration of stage A as BJD 2458455.02458462.5 ( 22 cycles) from the long, uniform superhump period and the amplitude. Considering a delay of start of our observations, the duration of stage $\mathrm{A}$ is somewhat longer. Judged from the ASAS-SN data, the duration of stage A superhumps is at most about 10 days. The growth time of superhumps is rather close to the case of WZ Sgetype DNe (Kato 2015).

Rebrightenings after the main superoutburst are also the intrinsic feature of WZ Sge-type DNe. The rebrightenings of ASASSN-18aan are similar to type-B rebrightening, which shows two or more repetitive short brightenings after a rapid decline of main superoutburst (Kato 2015; Kimura et al. 2016).

Most recently, the similarity of superoutbursts in long$P_{\text {orb }}$ SU UMa-type DNe to those in WZ Sge-type one was found in several objects (Antipin, Pavlenko 2002; Mroz et al. 2013; Kato et al. 2016; Kato et al. 2017; Kato et al. 2019a; Kato et al. 2019b; Kato 2020) Kato et al. (2016) proposed that such a behavior like WZ Sge-type superoutbursts is responsible for a difficulty in maintaining the tidal instability due to the weakness of tidal torque at the $3: 1$ resonance radius which lies close to the tidal truncation radius. Kato et al. (2019a) also found that the growth of 
the 3:1 resonance in systems with the stability border of the 3:1 resonance seems to be slow.

In the case of ASASN-18aan, the characteristics of the superoutburst are consistent with other long- $P_{\text {orb }} \mathrm{SU}$ UMa-type DNe. The similarity to the WZ Sge-type DNe in such a long- $P_{\text {orb }}$ SU UMa-type DNe would be a key for an investigation of the effect at the tidal truncation radius.

\subsection{Anomalously warm secondary}

A $0.18 \mathrm{M}_{\odot}$ main-sequence star will have a late $\mathrm{M}$ spectral type, dramatically cooler than the secondary spectrum we observe here. Mass loss evidently causes CV secondaries to depart from the main sequence mass-radiustemperature relation (Knigge et al. 2011), but the empirical donor sequence of Knigge (2006) shows CV secondaries near $P_{\text {orb }}=3.6 \mathrm{hr}$ are typically near type M5, with a scatter of a few subclasses. The secondary is much warmer than expected, for both its determined mass and the orbital period. ASASSN-18aan joins a small group of CVs known to have anomalously warm secondary stars. We summarized them in table 4.

An apparent enhancement of the sodium absorption line can be seen in the averaged spectrum after subtraction of a scaled spectrum of G9V-type star in figure 11. Enhanced sodium absorption is also seen in other warmerthan-expected systems, such as QZ Ser (Thorstensen et al. 2002a) and CSS J134052.0+151341 (Thorstensen 2013). Thorstensen et al. (2002a) suggested that this indicates a history of significant nuclear evolution of the secondary before mass transfer occurred; a side chain of hydrogen burning via CNO cycle, ${ }^{23} \mathrm{Ne}(p, \gamma){ }^{23} \mathrm{Na}$, leads to enhanced sodium. CV secondary stars in general depart somewhat from main-sequence mass-radius-luminosity relations, but the departures in these warm-secondary systems are much larger. Nuclear evolution prior to mass transfer can account for this for these gross discrepancies, as well, since the strong admixture of fused helium leads to a mean molecular weight very different from the main sequence. Evolutionary scenarios for these systems are explored briefly by Thorstensen et al. (2002b).

\section{Summary of Results}

We report photometry and spectroscopy of the eclipsing SU UMa-type DN ASASSN-18aan in its 2018 outburst and following quiescence. The superoutburst showed a slow growth rate of superhumps and rebrightenings, characteristics similar to that of WZ Sge-type DNe. We derived the orbital period to be $P_{\text {orb }}=0.149454(3) \mathrm{d}$ and confirmed this object has an extraordiarily long orbital period, for an SU
UMa-type DN, above the 2-3 hr period gap. ASASSN18 aan is a new member of the small class of long- $P_{\text {orb }} \mathrm{SU}$ UMa-type DNe. We also estimated the mass ratio using three different methods and derived nearly identical values; from the superoutburst we find $q=0.278(1)$. This is close to the upper limit for which the 3:1 resonance can cause the tidal instability (Whitehurst 1988; Murray et al. 2000).

By studying the eclipses we find that the disk radius at the onset of the superoutburst is close to or beyond the 3:1 resonance radius, and thus the superoutburst indeed can be caused by the tidal instability. This result suggests that other long- $P_{\text {orb }}$ SU UMa-type DNe also could be explained by the tidal instability model, without the need for other hypotheses. By considering long- $P_{\text {orb }}$ WZ Sge-type DNe (Wakamatsu et al. 2017), we further suggest that the tidal truncation radius is effective at larger mass ratios such as those found in SU UMa-type DNe, but in extremely low- $q$ systems it is no longer viable; in those cases the outbursting disk may reach the 2:1 resonance, which lies outside the tidal truncation radius.

One of our most striking results is that the secondary is much warmer than typical for CVs of this orbital period. Its spectral type is G9, while typical secondary stars at $P_{\text {orb }}=3.6 \mathrm{hr}$ are in the mid-M range. We also find indications of enhanced sodium abundance. Both these clearly indicate an unusual evolutionary history.

\section{Concluding remarks.}

ASASSN-18aan is an extremely unusual CV. The vast majority of SU UMa-type DNe have $P_{\text {orb }}$ less than about two hours, while the period we find is nearly twice that long. The period is derived from eclipses, and corroborated by a large modulation in the secondary star's radial velocities, so it is unquestionably orbital, and not due to some other phenomenon. In addition, the secondary star is highly anomalous; it is much hotter than typical CV secondaries in this period range, and is furthermore much less massive than its surface temperature would suggest. These gross peculiarities are very likely the consequence of mass transfer that began after the secondary star had undergone significant nuclear evolution; we are seeing, in effect, the stripped core of a much larger star. ASASSN-18aan joins a small number of similar systems already known.

Decades of careful study of superhumping systems have yielded a rich empirical picture of the superhump phenomena, which has spawned a correspondingly detailed body of theory. An anomalous sytem such as this one presents an opportunity for critical tests of such theories in relatively unexplored regions of parameter space. 
Table 4. List of CVs with anomalously warm secondary.

\begin{tabular}{|c|c|c|c|c|c|c|}
\hline Object & $P_{\text {orb }}{ }^{*}$ & $M_{1}^{\dagger}$ & $M_{2}^{\dagger}$ & $\begin{array}{l}\text { Spectral } \\
\text { type }\end{array}$ & $\begin{array}{l}\text { Spectral type pre- } \\
\text { dicted from } P_{\text {orb }}{ }^{\ddagger}\end{array}$ & References \\
\hline$(=1 \mathrm{RXS}$ & $0.044567(3)$ & 0.7 & 0.13 & $\mathrm{~K} 4 \pm 2$ & - & 1 \\
\hline \multicolumn{7}{|l|}{ J232953.9+062814) } \\
\hline QZ Ser & $0.0831612(11)$ & 0.7 & 0.125 & $\mathrm{~K} 4 \pm 2$ & M4-M5 & 2 \\
\hline SDSS J170213.26+322954.1 & $0.10008209(9)$ & 0.94 & 0.20 & M0 & M4 & 3 \\
\hline CSS J134052.0+151341 & $0.10213(8)$ & 0.7 & 0.5 & $\mathrm{~K} 4 \pm 2$ & M3-M4 & 4 \\
\hline ASASSN-13cl & $0.20219(8)$ & - & - & $\mathrm{K} 4 \pm 2$ & M3 & 5 \\
\hline ASASSN-14ho & $0.24315(10)$ & 1.00 & 0.28 & $\mathrm{~K} 4 \pm 2$ & M3 & 6 \\
\hline ASASSN-18aan & $0.149452(2)$ & 0.6 & 0.18 & G9 & M5 & $\begin{array}{l}\text { This } \\
\text { per. }\end{array}$ \\
\hline
\end{tabular}

*In units of day.

${ }^{\dagger}$ In units of $M_{\odot}$.

${ }^{\ddagger}$ Knigge (2006).

1. Thorstensen et al. (2002b); 2. Thorstensen et al. (2002a); 3. Littlefair et al. (2006); 4. Thorstensen (2013);

5. Thorstensen (2015); 6. Gasque et al. (2019)

\section{Acknowledgement}

This work was supported by the Optical and Near-infrared Astronomy Inter-University Cooperation Program and the Grants-in-Aid of the Ministry of Education. This research made use of the AAVSO Photometric All-Sky Survey (APASS), funded by the Robert Martin Ayers Sciences Fund and NSF AST-1412587. JRT thanks the MDM staff for observing support, and the Tohono O'odham Nation for allowing their mountain to be used for research into the sky that surrounds us all. S.S. was supported by the Slovak Research and Development Agency under the contract No. APVV-15-0458, by the Slovak Academy of Sciences grant VEGA No. 2/0008/17 and was partially supported by the Program of Development of M. V. Lomonosov Moscow State University "Leading Scientific Schools", project "Physics of Stars, Relativistic Objects and Galaxies". We are grateful to the All-Sky Automated Survey for Supernovae (ASAS-SN) project for detecting the superoutburst of ASASSN-18aan. YW is grateful to H. Kimura at Higashi-Hiroshima Observatory for collaboration on observation. YW deeply thanks the referee Dr. Elena P. Pavlenko for her quick review for my doctoral dissertation.

\section{Supporting Information}

Supplementary figure E1 and tables E1-E3 are reported in the online version.

\section{Appendix}

Our developed modeling method of eclipses during outbursts uses Metropolis algorithm (Metropolis et al. 1953), which is a kind of MCMC (for detail, see, e.g., Sharma (2017)).

We assumed observed magnitudes following a normal distribution with specific errors. The likelihood function is thus as follows:

$L=\prod_{i=1}^{n} \frac{1}{\sqrt{2 \pi \sigma_{i}^{2}}} \exp \left[-\frac{\left(x_{i}-y_{i}\right)^{2}}{2 \sigma_{i}^{2}}\right]$,

where $n, x_{i}, y_{i}$ and $\sigma_{i}$ are a number of data, observed flux, flux of the synthesized light curve and the photometric error at $i$-th data of the eclipse, respectively. We practically used a log-likelihood function. We set the map size at $201 \times 201$. The reason why we used this mesh value is that, as described in section 4.1, in this case, the occultation by the secondary grazes the center part of the accretion disk, which is a main part of the light source, and thus the synthesized light curve especially around the mid eclipse are strongly affected by the "coarseness" of the map. The smallest mesh value being able to ignore this effect is found to be 201. We set the photometric error at $\sigma_{i}=0.01$ in each $i$-th data.

New candidates of the parameters are independently generated from a normal distribution, i.e., the proposal distribution of each parameter is a normal distribution. We used non-informative prior distribution. Although there are some restrictions on each parameter (e.g., the inclination are estimated from the spectroscopic analysis), each parameter seemed to converge well. A width of step in each proposal distribution are decided to converge the ac- 
ceptance rate to be about 0.3 (Gelman et al. 1996).

We set the total number of iterations to be $2 \times 10^{4}$ and ignore the burn-in period to be $4 \times 10^{3}$. We calculated $95 \%$ credible intervals from the MCMC samples picked up every 50 iterations (i.e. thinning is 50) to avoid autocorrelations between iterations. We thus derived 320 samples as the random samples from the posterior distribution, which is what we want to estimate.

\section{References}

Antipin, S. V., \& Pavlenko, E. P. 2002, A\&A, 391, 565

Bąkowska, K., Olech, A., Pospieszyński, R., Martinelli, F., \& Marciniak, A. 2014, Acta Astron., 64, 337

Cleveland, W. S. 1979, J. Amer. Statist. Assoc., 74, 829

Davis, A. B., Shappee, B. J., Archer Shappee, B., \& ASASSN 2015, American Astron. Soc. Meeting Abstracts, 225, \#344.02

Eggleton, P. P. 1983, ApJ, 268, 368

Gaia Collaboration, et al. 2018, A\&A, 616, A1

Gasque, L. C., Hening, C. A., Hviding, R. E., Thorstensen, J. R., Paterson, K., Breytenbach, H., Motsoaledi, M., \& Woudt, P. A. 2019, AJ, 158, 156

Gelman, A., Roberts, G., \& Gilks, W. 1996, Bayesian statistics, 5,599

Green, G. M., Schlafly, E., Zucker, C., Speagle, J. S., \& Finkbeiner, D. 2019, ApJ, 887, 93

Green, G. M., et al. 2018, MNRAS, 478, 651

Hellier, C. 2001, PASP, 113, 469

Henden, A. A., Levine, S. E., Terrell, D., Smith, T. C., \& Welch, D. L. 2011, American Astronomical Society Meeting Abstracts \#218, Bulletin of the American Astronomical Society, 43, 126.01

Hessman, F. V., \& Hopp, U. 1990, A\&A, 228, 387

Hirose, M., \& Osaki, Y. 1990, PASJ, 42, 135

Ichikawa, S., \& Osaki, Y. 1994, PASJ, 46, 621

Jacoby, G. H., Hunter, D. A., \& Christian, C. A. 1984, ApJS, 56,257

Kato, T. 2015, PASJ, 67, 108

Kato, T. 2020, PASJ, 72, L2

Kato, T., et al. 2014a, PASJ, 66, 90

Kato, T., et al. 2014b, PASJ, 66, 30

Kato, T., Hambsch, F.-J., Monard, B., Nelson, P., Stubbings, R., \& Starr, P. 2019a, PASJ, 71, L4

Kato, T., Hambsch, F.-J., Oksanen, A., Starr, P., \& Henden, A. 2015, PASJ, 67,3

Kato, T., et al. 2009, PASJ, 61, S395

Kato, T., \& Osaki, Y. 2013, PASJ, 65, 115

Kato, T., et al. 2019b, PASJ, 71, L1

Kato, T., et al. 2016, PASJ, 68, L4

Kato, T., et al. 2017, PASJ, 69, L4

Kato, T., Uemura, M., Ishioka, R., Nogami, D., Kunjaya, C., Baba, H., \& Yamaoka, H. 2004, PASJ, 56, S1

Katysheva, N. A., \& Pavlenko, E. P. 2003, Astrophysics, 46, 114 Keenan, P. C., \& McNeil, R. C. 1989, ApJS, 71, 245

Kimura, M., et al. 2016, PASJ, 68, 55
Knigge, C. 2006, MNRAS, 373, 484

Knigge, C., Baraffe, I., \& Patterson, J. 2011, ApJS, 194, 28

Kochanek, C. S., et al. 2017, PASP, 129, 104502

Lin, D. N. C., \& Papaloizou, J. 1979, MNRAS, 186, 799

Littlefair, S. P., Dhillon, V. S., Marsh, T. R., \& Gänsicke, B. T. 2006, MNRAS, 371, 1435

Lubow, S. H. 1991, ApJ, 381, 259

Marsh, T. R., Horne, K., Schlegel, E. M., Honeycutt, R. K., \& Kaitchuck, R. H. 1990, ApJ, 364, 637

Martini, P., et al. 2011, PASP, 123, 187

Mennickent, R. E. 1995, A\&A, 294, 126

Metropolis, N., Rosenbluth, A. W., Rosenbluth, M. N., Teller, A. H., \& Teller, E. 1953, J. Chem. Phys., 21, 1087

Montgomery, M. M., Voloshina, I., Olenick, R., Meziere, K., \& Metlov, V. 2017, New Astron., 50, 43

Mroz, P., et al. 2013, Acta Astron., 63, 135

Murray, J., Warner, B., \& Wickramasinghe, D. 2000, New Astron. Rev., 44, 51

Nauenberg, M. 1972, ApJ, 175, 417

Nesci, R., Tuvikene, T., \& Gualandi, R. 2019, Open European Journal on Variable Stars, 196, 1

Osaki, Y., \& Meyer, F. 2002, A\&A, 383, 574

Paczyński, B. 1977, ApJ, 216, 822

Papaloizou, J., \& Pringle, J. E. 1977, MNRAS, 181, 441

Patterson, J., Jablonski, F., Koen, C., O'Donoghue, D., \& Skillman, D. R. 1995, PASP, 107, 1183

Pavlenko, E. P., et al. 2014, PASJ, 66, 111

Pavlenko, E. P., et al. 2018, Contributions of the Astronomical Observatory Skalnate Pleso, 48, 339

Pavlenko, E. P., et al. 2010, Astron. Rep., 54, 6

Pecaut, M. J., \& Mamajek, E. E. 2013, ApJS, 208, 9

Schreiber, M. R. 2007, A\&A, 466, 1025

Shappee, B. J., et al. 2014, ApJ, 788, 48

Sharma, S. 2017, Annual Review of Astronomy and Astrophysics, 55, 213

Smak, J., \& Waagen, E. O. 2004, Acta Astron., 54, 433

Smak, J. I. 2001, Acta Astron., 51, 279

Smak, J. I. 2006, Acta Astron., 56, 277

Stellingwerf, R. F. 1978, ApJ, 224, 953

Stolz, B., \& Schoembs, R. 1984, A\&A, 132, 187

Thorstensen, J. R. 2013, PASP, 125, 506

Thorstensen, J. R. 2015, PASP, 127, 351

Thorstensen, J. R., Alper, E. H., \& Weil, K. E. 2016, AJ, 152, 226

Thorstensen, J. R., \& Armstrong, E. 2005, AJ, 130, 759

Thorstensen, J. R., Fenton, W. H., Patterson, J. O., Kemp, J., Halpern, J., \& Baraffe, I. 2002a, PASP, 114, 1117

Thorstensen, J. R., Fenton, W. H., Patterson, J. O., Kemp, J., Krajci, T., \& Baraffe, I. 2002b, ApJ, 567, L49

Tonry, J., \& Davis, M. 1979, AJ, 84, 1511

Truss, M. R. 2007, MNRAS, 376, 89

Wakamatsu, Y., et al. 2017, PASJ, 69, 89

Whitehurst, R. 1988, MNRAS, 232, 35

Zorotovic, M., Schreiber, M. R., \& Gänsicke, B. T. 2011, A\&A, 536, A42 